\title{
A Collaborative Network Model for the Standards Community
}

\author{
Ovidiu Noran \\ Griffith University, Australia \\ O.Noran@griffith.edu.au
}

\begin{abstract}
Standards, as agreed-upon norms and requirements about systems, are essential pillars of enterprise and network operation and interoperability. However, standards themselves often display interoperability, inconsistency and overlap problems partly due to the custodian work groups' heterogeneity, the politics involved and limited communication and cooperation. This paper proposes and investigates the use of a Collaborative Network $(\mathrm{CN})$ model in the standards community so as to take advantage of the wealth of knowledge accumulated in this domain, the artefacts built and the lessons learned in practice. Following an introduction and a review of the current issues in standards development, the paper presents the specific features of the CNs and the Virtual Organisations (VOs) they would create in order to tackle standards creation and revision in an integrated and synergistic way. A case study is also used to describe a possible implementation of the $\mathrm{CN} / \mathrm{VO}$ model in practice and to illustrate the benefits of the proposed approach.
\end{abstract}

Keywords: Collaborative Networks, International Standards Organisation.

\section{Introduction}

Today's enterprises must permanently adapt to a competitive and ever-changing business environment. Continuous change processes support enterprise agility; however, they also have the potential to affect data, application and business processes interoperability at technical, syntactic, semantic and pragmatic levels. These problems can 'make or break' the affected parties and may occur within a single enterprise but also at company network level [1]. A potential solution for the conceptual and syntactic aspects of interoperability involves agreeing on and upholding standardised formats to overcome barriers (see [2], [3]). This in turn demands unambiguous, non-overlapping and interoperable standards as crucial enablers of enterprise and network agility and survival. Unfortunately however, standards are themselves often plagued by the above-mentioned problems, brought about by quasi-isolated creation and evolution. The result is low usability and end user confusion as to what standards to use and how, for a given task.

This paper proposes the use of a Collaborative Network (CN) [4] approach in order to tackle some of the root causes of the issues affecting standards - so that operational, competent and synergic teams can be formed to develop and revise standards in a consistent way, within an integrated and supportive environment. 


\section{Standards Development - Some Current Issues}

A simplistic image of standards is that of documented agreed-upon norms or requirements about systems of interest. The concepts discussed in this paper are widely applicable; however, the present scope is limited to technical standards developed by the International Standards Organisation (ISO) [5] (with input from other organisations such as IEEE [6] and INCOSE [7]), relevant to the proper (inter)operation of enterprises, CNs and Virtual Organisations (VOs) they form.

A large majority of the standards involve the work and consensus of experts that are typically volunteering their time and resources in the process. The use of standards is mandated by laws and many governmental and private organisations and agencies. Thus, to secure and manage projects, companies must abide by the standards specified by the clients and/or in legal documents. Standard administration and development is assigned to Work Groups (WGs) in Technical Committees (TCs) and Sub-Committees (SCs). Typically, the WGs have their own websites with access restricted to members. This results in low visibility between WGs and is likely to lead to the development of standards displaying coverage gaps, overlaps, redundancy and inconsistency.

ISO has developed a general vocabulary [5] and also usually standards have glossaries attached so as to formalise the terminology and improve interoperability; however, the vocabulary is generic and glossaries are often inconsistent across WGs working on related standards. As currently it is difficult to find and update other affected standards, changes to one standard do not automatically propagate to, or are checked for compliance with all other relevant standards.

Typically, several standards are required in order to set up and operate a project (whether cooperatively or not). While ISO maintains a website with the information relating to standards, it is often difficult for the average user to establish the standards required for a particular type of project. The free guides sometimes provided have a low usability and level of detail; in addition, they cannot cover and explain the use of every combination of standards as it will most likely be necessary. Terminology inconsistency, gaps, overlaps and interoperability deficiency of the standards that may have been selected using a guide add to the users' confusion and end up affecting all levels of enterprise(s) and network operation. There is some literature that explains the use of standards in more depth, albeit scarce and specialised (see the case of software development standards [8]).

There are currently several mechanisms within ISO to promote cooperative work and improve WG organisational interoperability. Thus, SCs hold yearly and halfyearly Plenary and Interim Meetings, where WG members meet to work but also socialise in events and ceremonies [9]. Study Groups (SWGs) recruit members across WGs in order to work on issues perceived as having common areas and 'liaisons' (members that belong to several WGs) are also used in order to facilitate information exchange. These approaches are a good baseline; however, they can be improved. For example, the meetings are too few to promote trust and cultural interoperability, especially in an environment where politics and lobbying for different agendas (other standards organisations, national bodies, major government contractors, etc) are an 
inherent part of decision-making. The SWGs creation occurs in a rather ad-hoc manner and the liaisons, while well-intended and hard-working, in the author's experience are often constrained by limited resources and authority.

As each standard has its own lifecycle, the mandatory review processes occur in an asynchronous way across ISO. Ideally, all other interested WGs should be aware of the proposed revisions to a standard and participate if necessary. Currently however, this is rather occurring on an irregular and anecdotal basis.

It also appears that currently, many TCs and SCs do not have a holistic view of the standards they develop and maintain and their potential impact on other standards. To the knowledge of the author, to date such views have only been attempted in an isolated and ad-hoc way. This lack of a 'big picture' further hinders the proper cooperation and consistency within and across SCs and TCs.

To summarise, the main problems are that a) members of various groups need to properly interoperate and collaborate in developing and revising standards and b) all relevant groups need to be involved in a project so that gaps, scattering and overlap is avoided so that the resulting standards are consistent in structure, vocabulary etc.

An analogy can be made here with commercial enterprises that come together in order to tackle projects requiring resources and knowledge beyond their own. Such enterprises typically set up (or join) CNs that allow them to get to know and trust each other. CNs act as 'breeding environments' who can promptly create VOs that successfully bid for projects, complete them and subsequently dissolve.

The following section attempts to explain how the $\mathrm{CN}$ concept can be applied to the universe of discourse of standards development.

\section{The Suitability of a Collaborative Network Model}

The $\mathrm{CN}$ paradigm, brought about by globalisation and ICT infrastructure progress, has evolved to become a scientific discipline [10]. The application of the $\mathrm{CN}$ principles nowadays is wide - in industry, aged care, medicine, education, defence, but also in areas such as social networking [11], or environmental sustainability and disaster management $[12,13]$. Interoperability (the lack of which is one of the root causes of standards development inconsistency and overlap problems) is paramount in the efficiency and survival of a $\mathrm{CN}$ - therefore it has been extensively researched (see [14] and many others). The intricate area of organisation and culture interoperability, very relevant to the standards community, has also been tackled (see e.g. [15]).

Adopting a $\mathrm{CN}$ approach for standards development would allow using all this wealth of $\mathrm{CN}$ and related interoperability knowledge. For example, to address the technical (such as infrastructure) and syntactic interoperability aspects, a shared 'online' intelligent repository (see e.g. [16]), capable of representing the standardsrelated information in various ways (including the life cycle context and interactive / 3D views as argued by Cleveland [17] and Gomes et al. [18]) would significantly benefit every type of group involved. The participants in a so-called ' $\mathrm{CN}$ for standards development' would also have the opportunity to address the organisational culture interoperability aspect by getting to know, understand (i.e. achieve semantic interoperability) each other and thus build trust and synergy. 


\subsection{Specific Features of the Collaborative Network and Virtual Organisations}

In order to be applied to standards development, the $\mathrm{CN}$ paradigm needs to be tailored to its specific requirements so it can effectively address the problems outlined in the previous sections. Thus, the commercial and competitive motivations of the typical $\mathrm{CN}$ participants are less present (perhaps in the companies that must use the standards produced and who seek to yield the standards to their advantage by lobbying WGs' members). Rather, the main motivation to enter what could be called a 'Standards Development Collaborative Network' (SDCN) would be to improve interoperability and efficiency of the WGs and other groups that must come together to create / revise standards. Such an SDCN could be formed at TC or SC level, comprising WGs, SWGs and other relevant external bodies (including individual experts - see Fig. 1).

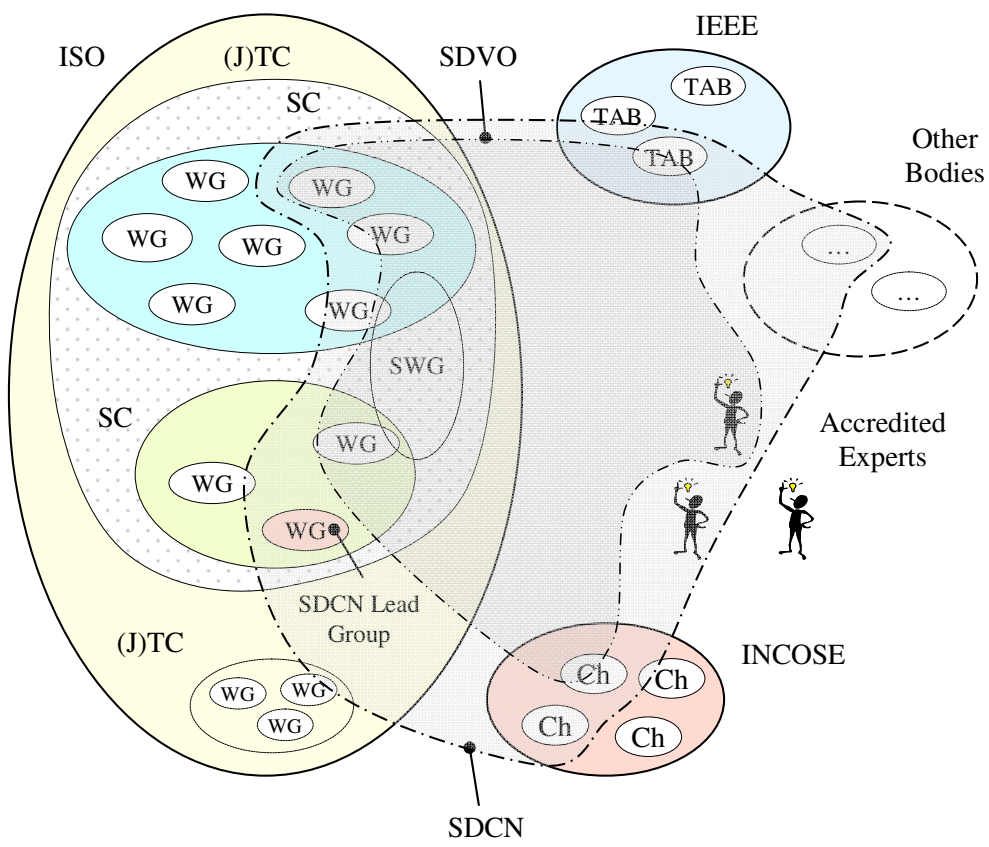

Legend: ISO: International Standards Organisation; IEEE = Institute of Electrical and Electronics Engineers; INCOSE = International Council on Systems Engineering; $(\mathrm{J}) \mathrm{TC}=($ Joint $)$ Technical Committee; SC=SubCommittee; WG = Work Group; SWG = Study Group; TAB = IEEE Technical Activities Board; SDCN = Standards Development Collab. Network; SDVO = Standards Development Virtual Org.; $\mathrm{Ch}=(\mathrm{INCOSE})$ Chapter; $-\cdot \cdot \cdot \cdot-\mathrm{SDCN}$ Boundary; $\quad-\cdot \cdot-\cdot=\mathrm{SDVO}$ Boundary

Fig. 1. Possible SDCN and SDVO structures

In a SDCN, the typical create / join / remain / leave the network decisions would be left at WG level with some guidance from the SC conveners and the ISO directives. Lessons learned from past activities are currently not effectively reused; they could be abstracted and stored in reference models contained in a structured repository made available to the entire SDCN and integrated into an ISO-wide expert system [16]. 
The 'Standards Development Virtual Organisations' (SDVOs) created would be similar to their commercial VO counterparts, except that they would not bid for a project but rather be assigned one such as a New Work Item (NWI) of standard creation, or a revision. The operating guidelines of the SDCN and SDVO would be set by the ISO rules. The 'lead partner', customary in commercial $\mathrm{CNs}$, could be represented here by the WG custodian of the new / revised standard.

Fig. 1 shows a simplistic view of an example SDCN and SDVO created by it. The network in this case reaches across several SCs and includes one SWG, WGs and structures from other interested organisations (in whole or in part according to the resources committed for the network) as well as individual experts accredited with ISO. The SDCN shown also has a 'lead group' that may be elected based on size, knowledge, resources, standards custody, etc. The SDVO is created as a subset of the SDCN with the mission to develop or revise one or several related standards.

\section{Case Study: Integrated Standard Development and Revision}

Systems and software systems engineering make use of two important standards, namely ISO 15288: Systems Life Cycle Processes [19] and ISO 12207: Software Life Cycle Processes [20]. The names suggest that ISO12207 is the specialisation of ISO15288 for the software domain. If that was true, ISO12207 should inherit and specialise the content of ISO15288, having identical shared definitions and inherited concepts such as life cycle phases, aspects, etc. Unfortunately, this is not the case as the two standards were developed by different WGs, at different times, with limited cross-consultation and input.

A harmonisation project was started to address these problems; however, it had a rather limited and erratic WG involvement and was beset by lobbying and politics. Work was discontinued after several years of efforts yielding disappointing results. Despite this outcome, valuable insight has been gained regarding a) the lack of a holistic image of the standards within the parent SC and b) the serious technical and organisational problems involved in the reconciliation of existing standards.

During the harmonisation efforts, a parallel attempt was made to use a framework [21] that was generic in nature, hence neutral and acceptable to 'mediate' between the two standards and identify gaps, overlaps and terminology inconsistency. This effort has led to the unsettling conclusions that c) the mediating framework itself and several related standards also displayed terminology inconsistencies with the two standards in question and d) the custodian WGs were not fully aware of the problem.

\subsection{Application of the Proposed Collaborative Network Approach}

In order to address the above-mentioned conclusions and problems, it is proposed to create an SDCN as shown in Fig. 1, supported by an intelligent shared repository such as described in [16]. The call for SDCN creation can be broadcast at Plenary / Interim Meetings. WGs and other bodies (IEEE, INCOSE, etc) shall identify themselves as 
stakeholders / custodians of the standards and start working together to achieve proper interoperability in all relevant aspects (semantic in particular). The SDCN will allocate members for the SDVO that will tackle the envisaged project. In this case, the SDCN members may be WG7, WG10, SWG5 from JTC1/SC7, WG1 from TC184/SC5, Technical Activities Board (TAB) members from IEEE and US / EU Chapter members from INCOSE. The SDVO members would be members of WG7, WG10, all SWG5 and interested IEEE TAB and INCOSE US Chapter.

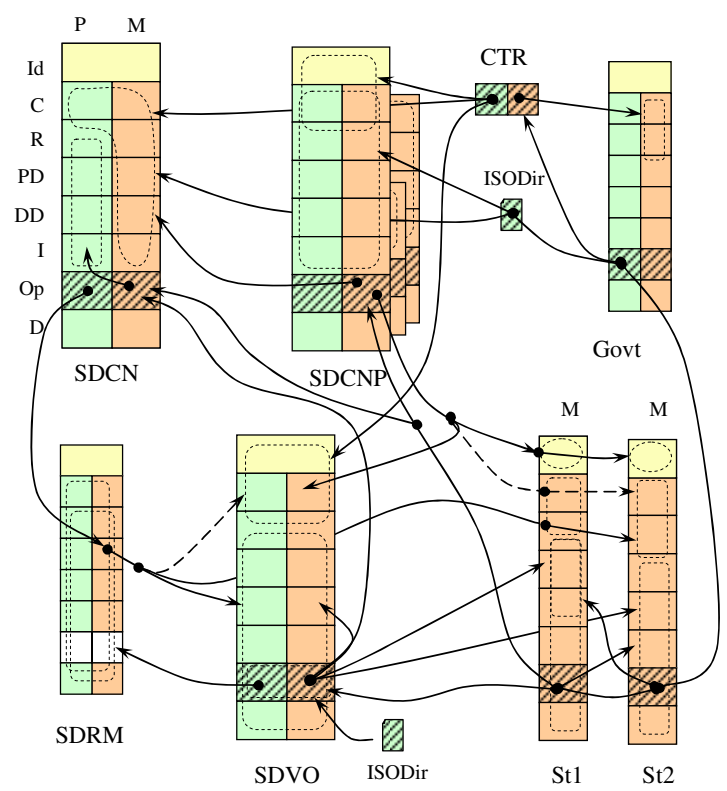

\begin{tabular}{|c|c|}
\hline & Legend: \\
\hline SDCN: & $\begin{array}{l}\text { Standards Development } \\
\text { Collaborative Network }\end{array}$ \\
\hline SDCNF & $\begin{array}{l}\text { P:Standards Development } \\
\text { Collaborative Network } \\
\text { Participants }\end{array}$ \\
\hline ISODir & : ISO Directives \\
\hline SDRM & $\begin{array}{l}\text { Standards Development } \\
\text { Reference Model(s) }\end{array}$ \\
\hline SDVO & $\begin{array}{l}\text { : Standards Development } \\
\text { Virtual Organisation }\end{array}$ \\
\hline Gvt & : Government \\
\hline $\mathrm{St}(\mathrm{x})$ & : Target Standards \\
\hline CTR & : Contractors (e.g. defence) \\
\hline ZDDA & $\begin{array}{l}\text { Operation phase, Production } \\
\text { \& Management }\end{array}$ \\
\hline$\theta$ & : Operation Phase, Production \\
\hline & $\begin{array}{l}\text { : Possible scenario } \\
\text { : Area covered }\end{array}$ \\
\hline
\end{tabular}

Life cycle phases: $\mathbf{I d}=$ Identification; $\mathbf{C}=$ concept $\mathbf{R}=$ requirements, $\mathbf{P D}=$ preliminary design, $\mathbf{D D}=$ detailed design, $\mathbf{I}=$ implementation, $\mathbf{O p}=$ operation, $\mathbf{D}=$ decommissioning. Other aspects: $\mathbf{P}=$ Production $/$ Service, $\mathbf{M}=$ management

Fig. 2. Life cycle-based model of SDCN and SDVO creation / operation

As all participant groups, organisations and entities evolve, the interactions that take place between the participants must be considered and represented in a life cycle context. In this paper, we use a modelling formalism derived from the reference used to mediate between the standards within the cased study, namely ISO15704 Annex A. This artefact contains a framework whose modelling framework (MF), called the Generalised Enterprise Reference Architecture (GERA), contains a rich repository of aspects including life cycle, management, organisation, human, decision, etc (see [21] for details). Fig. 2 shows the interactions between the participants in the proposed $\mathrm{CN}$ model using a GERA MF-based formalism featuring only the life cycle and management/operations viewpoints. The arrows represent the interactions between the participants in the context of their life cycles. Details have been omitted in the attempt to emphasize the most important features of the proposed model, as further described.

As shown in the figure, the SDCN is created by participants (the arrows from SDNCP to SDCN's Concept to Implementation life cycle phases). The SDCN then 
creates SDVOs as required in order to create or revise standards (St) (the arrows from SDCN to SDVO's Requirements to Decommissioning life cycle phases).

Beyond this basic interpretation however, this kind of representation also allows to show more intricate and essential facts that help stakeholders understand, adapt and better manage the standard development endeavour. Thus, it can be seen (arrows from CTR to one of the SDCNPs) that SDCN participants are lobbied or even created by some end users (e.g. larger companies that are required to use the standards by government (Govt) and laws, or other bodies who are interested in influencing and/or adopting the standards) and operate in accordance to the ISO Directives.

The SDCN and the SDVO created by it have a certain level of agility (arrows in SDCN and SDVO from Operation phase to their own upper life cycle phases) - i.e. they can 'redesign themselves' to a certain degree to achieve some stability in the face of changes in the environment (laws, other standards, WGs, etc). The figure also shows that the extent of the standards designed by the SDVO can vary. Some phases, e.g. identifying the need for a standard and defining the concepts underlying the standards may come straight from the SDCN participants (arrows from SDCNP to upper phases of St1 and St2). The same applies for the SDVO itself.

Importantly, standards influence each other's development (arrows from St1 to St2 and vice versa). This aspect must be detailed in additional models and implemented in the supporting structured repository so that changes to a standard can propagate to all related standards via their respective custodian WGs and any other SDVOs in charge.

The artefacts built and lessons learned during SDCN and SDVO creation and operation can be abstracted in reference models stored in a structured repository as previously described (arrows from SDCN, SDVO to SDRM).

\section{Conclusions and Further Work}

Standards development is a complex endeavour that is made possible by volunteers having a large variety of backgrounds and cultures. While notable results have been achieved, the standards community could further benefit from adopting a holistic and life cycle-based view of the standards and groups involved, addressing the politics and lack of trust and bridging organisational and geographical culture gaps that trigger counter-productive semantic barriers between participants.

The proposed model is based on SDCNs (supported by an integrated repository) who can timely and optimally select participants and build a SDVO for the required task. The work accomplished by SDVOs is broadcast and visible to all relevant stakeholders. Importantly, in this model the principles and vocabulary reflecting the SDCN participants' knowledge is inherently consistent across all products created or revised by the SDVOs. Redundant work is avoided, inconsistencies are eliminated, conflict and politics are minimised and efficiency and cooperation are improved.

There are also a number of caveats to this proposal. Firstly, the proposed CN model implies changes to ISO's organisational culture. Secondly, the creation and operation of the SDCNs and SDVOs must be regulated in the ISO directives to allocate proper authority and responsibility. And thirdly, a supporting infrastructure 
mirroring and enabling the proposed model must be implemented and accepted (and thus, actually used) by the work groups. Facilitating and modelling these change processes and artefacts constitutes the focus of further research and work in this area.

\section{References}

1. EI2N \& CoopIS. Interoperability Issues in Collaborative Information Systems (Presentation to the Plenary) (2010),

http://tc.ifac-control.org/5/3/events/ei2n2010-

folder/ei2n2010-presentations-reports-and-

conclusions/EI2N_2010_Interoperability_Issues_in_Collaborati

ve_Information_Systems.pdf/at_download/file

2. Chen, D.: Framework for Enterprise Interoperability (2006),

http: / / www. fines-cluster. eu/fines/jm/Download-document/53-

Framework-for-Enterprise-Interoperability-Chen.html

(cited July 2011)

3. Noran, O., Bernus, P.: Effective Disaster Management: An Interoperability Perspective. In: Meersman, R., Dillon, T., Herrero, P. (eds.) OTM-WS 2011. LNCS, vol. 7046, pp. 112121. Springer, Heidelberg (2011)

4. Camarinha-Matos, L., Afsarmanesh, H.: Collaborative Networks: A new scientific discipline. Journal of Intelligent Manufacturing 16, 439-452 (2005)

5. ISO. ISO/IEC Guide 2:Standardization and related activities - General vocabulary (2004),

http://www.iso.org/iso/iso_iec_guide_2_2004.pdf (cited 2011)

6. IEEE. Institute of Electrical and Electronics Engineers - Technical Activities Board Ops Manual (2012),

http: / /www. ieee.org/about/volunteers /

tab_operations_manual.pdf

7. INCOSE. International Council on Systems Engineering - About INCOSE (2012), http: / / www . incose.org/about/index.aspx (cited 2012)

8. Moore, J.M.: The Road Map to Software Engineering: A Standards-Based Guide, 1st edn. Software Engineering Standards 2006. Wiley-IEEE Computer Society $\operatorname{Pr}(2006)$

9. Portes, A.: Social Capital: Its Origins and Applications in Modern Sociology. Annual Review of Sociology 24, 1-24 (1998)

10. Camarinha-Matos, L., et al.: Collaborative networked organizations - Concepts and practice in manufacturing enterprises. Computers and Industrial Engineering 57(1), 46-60 (2009)

11. Badr, Y., Faci, N., Maamar, Z., Biennier, F.: Multi-level Social Networking to Enable and Foster Collaborative Organizations. In: Camarinha-Matos, L.M., Pereira-Klen, A., Afsarmanesh, H. (eds.) PRO-VE 2011. IFIP AICT, vol. 362, pp. 3-10. Springer, Heidelberg (2011)

12. Noran, O.: Towards an Environmental Management Approach for Collaborative Networks. In: Camarinha-Matos, L., Boucher, X., Afsarmanesh, H. (eds.) Collaborative Networks for a Sustainable World, pp. 17-24. Springer, Berlin (2010)

13. Noran, O.: Towards a Collaborative Network Paradigm for Emergency Services. In: Camarinha-Matos, L.M., Pereira-Klen, A., Afsarmanesh, H. (eds.) PRO-VE 2011. IFIP AICT, vol. 362, pp. 477-485. Springer, Heidelberg (2011) 
14. ATHENA State of the art of Enterprise Modelling Techniques and Technologies to Support Enterprise Interoperability. Deliv D.A1.1.1 2(004), http: / /www . athena-ip.org (cited March 30, 2011)

15. Whitman, L., Panetto, H.: The Missing Link: Culture and Language Barriers to Interoperability. Annual Reviews in Control 30(2), 233-241 (2006)

16. Noran, O.: Towards a Sustainable Interoperability of Standards. In: 14th IFAC Symposium on Information Control Problems in Manufacturing, Bucharest, Romania (2012)

17. Cleveland, W.S.: Visualizing Data. Hobart Press (2008)

18. Gomes, A., Maneschy, M.C.: Communication and Power in Collaborative Networks: The Hypothesis of Technology as Confidence Enhancer. In: Camarinha-Matos, L.M., PereiraKlen, A., Afsarmanesh, H. (eds.) PRO-VE 2011. IFIP AICT, vol. 362, pp. 19-26. Springer, Heidelberg (2011)

19. ISO/JTC1/SC7, ISO/IEC15288: Information Technology - Life Cycle Management System Life Cycle Processes (2008)

20. ISO/JTC1/SC7, ISO/IEC12207 (Amd2): Standard for Information Technology - Software life cycle processes (2008)

21. ISO/IEC, Annex A: GERAM, in ISO/IS 15704:2000/Amd1:2005: Industrial automation systems - Requirements for enterprise-reference architectures and methodologies (2005) 